\title{
Single-molecule Tracking Reveals Multi-state Dynamics of a Bacterial DNA Methyltransferase in Vivo
}

\author{
Ziyuan Chen, Taylor Nye, Lyle Simmons and Julie Biteen \\ University of Michigan, Ann Arbor, Michigan, United States
}

Single-molecule super-resolution microscopy enables quantitative tracking of molecules with great sensitivity and precision. By visualizing individual, isolated molecules tagged with fluorescent labels and then fitting the emission pattern, molecular positioning and trajectories can be acquired with high spatial and temporal resolution. We have been developing approaches to measure these dynamics in living bacterial cells and to accurately analyze and understand the rich data provided by single-molecule tracking.(1)

Measuring the dynamics of target molecules helps us to understand their function. Brownian diffusion theory has been widely used to determine diffusion coefficients and thereby characterize the dynamics of single-molecule trajectories. However, not all subcellular motions can be approximated as free diffusion. For instance, anomalous diffusion theory has been applied to explain non-Brownian behaviors that arise due to crowding and confinement in the subcellular or environment or upon the interaction between different molecules.(2, 3) Overall, carefully measuring subcellular single-molecule tracks and then analyzing the data in the appropriate framework can provide mechanistic insight into important problems in cell biology.

Here, we use super-resolution microscopy and single-molecule tracking to determine the localization and dynamics of the DNA methyltransferase DnmA in the Gram-positive bacterium Bacillus subtilis. The genomes of all organisms harbor epigenetic base modifications, which include N4-methylcytosine (m4C), C5-methylcytosine (m5C), and N6-methyladenine (m6A). These important base modifications are catalyzed by a DNA methyltransferase. The newly characterized DnmA is responsible for all the detectable m6A methylation in the genome of B. subtilis. Our single-molecule investigations of the dynamics of DnmA-PAmCherry in vivo will provide crucial information to elucidate the methylation process and the regulation mechanism of this DNA modifier.

We analyze our data under a nonparametric Bayesian statistics analytical framework for single particle trajectories,(4) and we find several different dynamical behaviors for DnmA. The multi-state dynamics of this DNA-binding protein likely involve a combination of 1-D sliding, hopping, and 3-D diffusion; uncovering this set of mobility states promises to reveal if the DnmA target searching is facilitated by 1$\mathrm{D}$ sliding and will further provide the translocation and dissociation rates of DnmA. We have also extended our analysis beyond the Brownian diffusion framework by developing a feature-based trajectories classifier to determine the anomalous diffusion model for DnmA based on our DnmA trajectories. We notice different anomalous diffusion behaviors for DnmA in different dynamics states. For instance, the slowest diffusing molecules also tend to be the most confined and show apparent subdiffusive behavior. In addition to providing mechanistic insight into the biological function of DnmA and about the intracellular environment, our results demonstrate that single-molecule tracking can be used to quantitatively investigate non-Brownian protein dynamics in vivo.

\section{References}


1. H. H. Tuson, J. S. Biteen, Unveiling the Inner Workings of Live Bacteria Using Super-Resolution Microscopy. Anal. Chem. 87, 42-63 (2015).

2. A. S. Hansen, A. Amitai, C. Cattoglio, R. Tjian, X. Darzacq, Guided nuclear exploration increases CTCF target search efficiency. Nat. Chem. Biol. (2019), doi:10.1038/s41589-019-0422-3.

3. J.-H. Jeon, M. Javanainen, H. Martinez-Seara, R. Metzler, I. Vattulainen, Protein Crowding in Lipid Bilayers Gives Rise to Non-Gaussian Anomalous Lateral Diffusion of Phospholipids and Proteins. Phys. Rev. X. 6, 021006 (2016).

4. J. D. Karslake, E. D. Donarski, S. A. Shelby, L. M. Demey, V. J. DiRita, S. L. Veatch, J. S. Biteen, "SMAUG: Analyzing single-molecule tracks with nonparametric Bayesian statistics" (preprint, Biophysics, 2019), doi:10.1101/578567. 